\title{
Gödel Type Metrics in Three Dimensions
}

\author{
Metin Gürses \\ Department of Mathematics, Faculty of Sciences \\ Bilkent University, 06800 Ankara - Turkey
}

November 1, 2018

\begin{abstract}
We show that the Gödel type Metrics in three dimensions with arbitrary two dimensional background space satisfy the Einstein-perfect fluid field equations. There exists only one first order partial differential equation satisfied by the components of fluid's velocity vector field. We then show that the same metrics solve the field equations of the topologically massive gravity where the two dimensional background geometry is a space of constant negative Gaussian curvature. We discuss the possibility that the Gödel Type Metrics to solve the Ricci and Cotton flow equations. When the vector field $u^{\mu}$ is a Killing vector field we finally show that the stationary Gödel Type Metrics solve the field equations of the most possible gravitational field equations where the interaction lagrangian is an arbitrary function of the electromagnetic field and the curvature tensors.
\end{abstract}




\section{Introduction}

In three dimensions there are several attempts to find exact solutions of Einstein and Topologically Massive Gravity field equations [1]-[4]. In these efforts authors usually start with a specific ansatz for the spacetime metrics. It seems that the Gödel type metrics [5]-[7] will be very convenient and practical in searching solutions of the field equations in three dimensions.

In a $D$ dimensional spacetime the Gödel type metrics are defined by

$$
g_{\mu \nu}=h_{\mu \nu}-u_{\mu} u_{\nu}
$$

where $h_{\mu \nu}$ is the metric of a $D-1$-dimensional locally Euclidean Einstein space with $h_{\mu \nu} u^{\mu}=0$ and $u_{\mu}$ is a unit timelike vector field with $u^{\mu}=-\frac{1}{u_{0}} \delta_{0}^{\mu}$. We studied these metrics when $u_{0}$ is a constant in [5] and when $u_{0}$ is not constant in [6]. Although, in these works our approach was independent of the dimension of the spacetime we focused our attention to the cases $D>3$ in much detail. In these works we obtained exact solutions of various supergravity theories in various dimensions. In [5] since $u_{0}$ was considered to be a constant our solutions contain no dilaton field. If $u_{0}$ is not a constant it plays the role of the dilaton field. In [6] we found exact solutions of the supergravity theories with dilaton. In [7] we studied the closed timelike curves in Gödel type metrics and showed that when the vector field $u_{\mu}$ is also a Killing vector of the spacetime geometry then there always exist closed timelike or null curves in Gödel type spacetimes.

In this work we shall consider the Gödel type metrics in three dimensions with $u_{0}$ constant (or $g_{00}$ is a constant). The case with $u_{0}$ is not a constant will be discussed later. There are several interesting properties of the spacetime geometry in three dimensions. In two dimensions since the Ricci tensor is proportional to the metric then the metrics of any three dimensional space-

time is of Gödel type. In three dimensions energy momentum tensor of a Maxwell field of the vector filed $u_{\mu}$ is equivalent to the energy momentum tensor of a perfect fluid with stiff equation of state. With these properties we show in Section 3 that any Gödel type metrics in three dimensions satisfy the Einstein-Perfect fluid field equations. Using the result of Section 3 we show in Section 4 that Gödel type metrics in three dimensions satisfy the field equations of the topological massive gravity (TMG) provided that the two dimensional space is a space of constant curvature. We find all possible 
Gödel type solutions of TMG and show that our previous solution [1] of TMG is a special case. The Ricci and Cotton tensors for the Gödel type metrics take very simple forms which attracts us to consider the corresponding flow equations. We study the Ricci and Cotton flow equations in Section 5. In the last Section we construct a closed tensor algebra which enables the Gödel type metrics to solve the field equations of a most general Lagrange function of metric, Ricci, curvature and the antisymmetric Maxwell tensor field and their covariant derivatives at all order.

\section{Gödel Type Metrics in General Relativity}

Let $u^{\mu}=-\frac{1}{u_{0}} \delta_{0}^{\mu}$ be a timelike vector with $u_{0}=$ constant, in $D$ dimensional spacetime $M$ and $h_{\mu \nu}$ be the metric of $D-1$ dimensional Euclidean space such that $u^{\mu} h_{\mu \nu}=0$. Gödel type of metrics are defined by [5]

$$
g_{\mu \nu}=h_{\mu \nu}-u_{\mu} u_{\nu}
$$

Let us define an antisymmetric tensor $f_{\mu \nu}$ as

$$
f_{\alpha \beta}=u_{\beta, \alpha}-u_{\alpha, \beta} .
$$

The Christoffel symbol corresponding to the metric (1) is

$$
\Gamma_{\alpha \beta}^{\mu}=\gamma_{\alpha \beta}^{\mu}+\frac{1}{2}\left(u_{\alpha} f_{\beta}^{\mu}+u_{\beta} f_{\alpha}^{\mu}\right)-\frac{1}{2}\left(u_{\alpha \mid \beta}+u_{\beta \mid \alpha}\right) u^{\mu},
$$

where a vertical stroke denotes covariant derivative with respect to the Christoffel symbol $\gamma_{\alpha \beta}^{\mu}$ and a semicolon or nabla $\nabla$ will denote covariant derivative with respect to the Christoffel symbol $\Gamma_{\alpha \beta}^{\mu}$. It is easy to show that

$$
u^{\alpha} \partial_{\alpha} u_{\beta}=0, \quad u^{\alpha} f_{\alpha \beta}=0 .
$$

Then

$$
\dot{u}^{\mu}=u^{\alpha} u_{; \alpha}^{\mu}=0 .
$$

It is a property of the Gödel type of geometries that the vector field $u^{\mu}$ is also a timelike Killing vector field of the spacetime geometry $(M, g)$ and hence we have 


$$
f_{\alpha \beta}=2 u_{\beta ; \alpha} .
$$

We define the current vector $j_{\mu}$ corresponding to the antisymmetric tensor field $f_{\mu \nu}$ as

$$
j_{\mu} \equiv f_{\mu \mid \alpha}^{\alpha}=\nabla_{\alpha} f_{\nu}^{\alpha}-\frac{1}{2} f^{2} u_{\nu}
$$

where $f^{2}=f_{\mu \nu} f^{\mu \nu}$.

It is now straightforward to prove the following Proposition [5]

Proposition 1. Let $(M, g)$ be a stationary spacetime with the Gödel type metric (1). Let $h_{\mu \nu}$ be the metric tensor of D-1-dimensional locally Euclidean space, then the Einstein tensor becomes

$$
\begin{aligned}
G_{\mu \nu}= & r_{\mu \nu}-\frac{1}{2} h_{\mu \nu} r+\frac{1}{2} T_{\mu \nu}^{f}+\frac{1}{2}\left(j_{\mu} u_{\nu}+j_{\nu} u_{\mu}\right)+ \\
& \left(\frac{1}{4} f^{2}+\frac{1}{2} r\right) u_{\mu} u_{\nu}-\frac{1}{2}\left(u^{\alpha} j_{\alpha}\right) g_{\mu \nu},
\end{aligned}
$$

where $T_{\mu \nu}^{f}$ denotes the Maxwell energy-momentum tensor for $f_{\mu \nu}, r_{\mu \nu}$ is the Ricci tensor of $\gamma_{\alpha \beta}^{\mu}$. The Ricci scalar is obtained as

$$
R=r+\frac{1}{4} f^{2}+u^{\mu} j_{\mu}
$$

where $r$ denotes the Ricci scalar of $r_{\alpha \beta}$.

The above Proposition gives the Einstein tensor of (1) without any conditions. In order to have a physical energy momentum distribution we assume that $D-1$-dimensional space is an Einstein space (a vacuum space with a cosmological constant) and the current vector field $j_{\mu}$ vanishes everywhere. Then we have

Proposition 2. Let $(M, g)$ be a stationary spacetime geometry with the Gödel type metric (1). Let $h_{\mu \nu}$ be the metric tensor of the D-1-dimensional Einstein space, $r_{\mu \nu}=\frac{r}{D-1} h_{\mu \nu}$ and let $j_{\mu}=0$. Then the metric $g_{\mu \nu}$ satisfies the Einstein field equations with a charged fluid

$$
G_{\mu \nu}=\frac{1}{2} T_{\mu \nu}^{f}+(p+\rho) u_{\mu} u_{\nu}+p g_{\mu \nu}
$$


with

$$
\begin{aligned}
\nabla_{\mu} f^{\mu \nu} & =\frac{1}{2} f^{2} u^{\nu}, \\
p & =\frac{(3-D)}{2(D-1)} r \\
\rho & =\frac{1}{4} f^{2}+r .
\end{aligned}
$$

Here $p$ is the pressure and $\rho$ is the energy density of the charged perfect fluid.

Here the signs of the fluid pressure and the fluid energy density depends (in particular for $D \geq 3$ ) on the sign of the Ricci scalar of the $D-1$ dimensional Euclidean space with metric $h_{\mu \nu}$.

Corollary 3. If $h_{\mu \nu}$ is the metric of a Ricci flat space then the energy momentum distribution for the Einstein field equations for the metric $g_{\mu \nu}$ becomes charged dust,i.e.,

$$
G_{\mu \nu}=\frac{1}{2} T_{\mu \nu}^{f}+\frac{1}{4} f^{2} u_{\mu} u_{\nu}
$$

provided $f$ satisfies the equation

$$
f_{\beta \mid \alpha}^{\alpha}=0
$$

and $T^{f}$ is the Maxwell energy momentum tensor for the antisymmetric tensor $f$

$$
T_{\mu \nu}^{f}=f_{\mu \alpha} f_{\nu}^{\alpha}-\frac{1}{4} f^{2} g_{\mu \nu}
$$

where $f^{2}=f^{\alpha \beta} f_{\alpha \beta}$. Maxwell's equations (15) can also be written as (11)

Hence Gödel type metrics (1) satisfy the Einstein field equations with charged dust distributions where the only field equations are the Maxwell equations (15) or (??) and the Ricci flat equations for $h_{\mu \nu}$. There is no electric field $\left(u^{\mu} f_{\mu i}=f_{0 i}=0\right)$, only the magnetic field exists. We have the gauge freedom

$$
u_{\mu}^{\prime}=u_{\mu}+\partial_{\mu} \Omega
$$


where $\Omega$ is a function satisfying the condition (recall that $u^{\prime \mu}=-\frac{\delta_{0}^{\mu}}{u_{0}^{\prime}}, \quad u^{\mu}=$ $\left.-\frac{\delta_{0}^{\mu}}{u_{0}}\right)$

$$
u_{0}^{\prime}=u_{0}+\partial_{0} \Omega
$$

Both $u_{0}$ and $u_{0}^{\prime}$ are constants. For the stationary spacetime, which is the case in this work, we have $\Omega$ not depending on $x^{0}$ and $u_{0}^{\prime}=u_{0}$ (constant) but this leads to constant $\Omega$.

$$
u^{\prime \mu}=u^{\mu}+g^{\mu \nu} \Omega_{, \nu}
$$

or

$$
\frac{\delta_{0}^{\mu}}{u_{0}^{\prime}}=\frac{\delta_{0}^{\mu}}{u_{0}}-g^{\mu \nu} \Omega_{, \nu}
$$

since $u_{0}^{\prime}=u_{0}$ then $\Omega=$ constant. Hence we have the following Proposition

Proposition 4. The only gauge transformation

$$
h_{\mu \nu}^{\prime}=h_{\mu \nu}, \quad u_{\mu}^{\prime}=u_{\mu}+\partial_{\mu} \Omega
$$

keeping the stationary Gödel type metric invariant is the one with constant $\Omega$.

In this work we only considered the case where $u_{0}$ is a non vanishing constant. In [6] we have studied the Gödel type metrics when $u_{0}$ is not a constant. In this case, the proposed metric yields exact solutions to the various theories with a dilaton field.

\section{Gödel Type Metrics in Three Dimensions}

In three dimensions Gödel type of metrics have very interesting properties. All three dimensional metrics can be written as a Gödel type of metric with a non-constant $u_{0}$.

Proposition 5. All metrics of the spacetime geometry are of Gödel type with a non-constant $u_{0}$. Any three dimensional metric can be written as follows: 


$$
\begin{aligned}
d s^{2}= & -P^{2}\left(d x^{0}\right)^{2}+2 M d x^{0} d x^{1}+2 N d x^{0} d x^{2}+ \\
& Q^{2}\left(d x^{1}\right)^{2}+2 L d x^{1} d x^{2}+R^{2}\left(d x^{2}\right)^{2} \\
= & -P^{2}\left(d x^{0}-\frac{M}{P^{2}} d x^{1}-\frac{N}{P^{2}} d x^{2}\right)^{2}+\left(\frac{M^{2}}{P^{2}}+Q^{2}\right)\left(d x^{1}\right)^{2}+ \\
& 2\left(L+\frac{M N}{P^{2}}\right) d x^{1} d x^{2}+\left(\frac{N^{2}}{P^{2}}+R^{2}\right)\left(d x^{2}\right)^{2}
\end{aligned}
$$

where $P, M, N, Q, L, R$ are functions of $x^{0}, x^{1}$ and $x^{2}$. Then the last form (21) is of Gödel type (1) with

$$
\begin{aligned}
u_{\mu} d x^{\mu}= & P\left(d x^{0}-\frac{M}{P^{2}} d x^{1}-\frac{N}{P^{2}} d x^{2}\right) \\
h_{\mu \nu} d x^{\mu} d x^{\nu}= & \left(\frac{M^{2}}{P^{2}}+Q^{2}\right)\left(d x^{1}\right)^{2}+2\left(L+\frac{M N}{P^{2}}\right) d x^{1} d x^{2}+ \\
& \left(\frac{N^{2}}{P^{2}}+R^{2}\right)\left(d x^{2}\right)^{2}
\end{aligned}
$$

Hence $u_{0}=P$ which is not a constant in general and $h$ is the metric of a two dimensional locally Euclidean space.

Corollary 6. When $(M, g)$ is stationary and $u_{0}=$ constant then metric functions depend on $x^{1}$ and $x^{2}$, and $P=$ constant

Another interesting property of three dimensions is that any antisymmetric tensor field $f_{\mu \nu}$ can be expressed as $\epsilon_{\mu \nu \alpha} v^{\alpha}$ where $v^{\alpha}$ is any vector field. Since $u_{0}$ is constant and $u_{\alpha, 0}=0$ (stationarity) then $u^{\alpha} f_{\alpha \beta}=0$. This implies that $v^{\alpha}$ is proportional to $u^{\alpha}$. Hence we have the following Proposition:

Proposition 7. The antisymmetric tensor $f_{\mu \nu}$ can be expressed as

$$
f_{\mu \nu}=2 w \eta_{\mu \nu \alpha} u^{\alpha}
$$

where $w$ is an arbitrary function and $\eta_{\mu \nu \alpha}=\sqrt{|g|} \epsilon_{\mu \nu \alpha}$. Here $\epsilon_{\mu \nu \alpha}$ is the totally antisymmetric Levi-Civita tensor. Hence from (7) we have

$$
\nabla_{\mu} u_{\nu}=w \eta_{\mu \nu \alpha} u^{\alpha}
$$


Taking the divergence of (23) we obtain

$$
\nabla_{\mu} f^{\mu \nu}=\frac{1}{2} f^{2} u^{\nu}+2 w_{, \alpha} \eta^{\alpha \nu \mu} u_{\mu}
$$

where $w^{2}=\frac{1}{8} f^{2}$. This leads to the following result:

Proposition 8. The above equations (25) imply that in three dimensions the Maxwell equations (??) are satisfied if and only if $w$ or $f^{2}=$ constant.

In three dimensions due to the property (23) the energy momentum tensor of $f_{\mu \nu}$ becomes the energy momentum tensor of a perfect fluid with $p=\rho$ equation of state.

Proposition 9. In three dimensions due to the property (23) the energy momentum tensor corresponding to the antisymmetric tensor field $f_{\mu \nu}$ reduces to

$$
T_{\mu \nu}^{f}=\frac{1}{2} f^{2} u_{\mu} u_{\nu}+\frac{1}{4} f^{2} g_{\mu \nu}
$$

where the the energy density and the pressure are respectively given by

$$
\rho=\frac{1}{4} f^{2}, \quad p=\frac{1}{4} f^{2} .
$$

Hence the we have the stiff equation of state $p=\rho$.

Then any stationary spacetime metric in three dimensions with $f^{2}=$ constant satisfies the Einstein perfect fluid field equations. We state this as the next proposition which will be used later for different purposes.

Proposition 10. Let $D=3$ in Proposition 2 and use Proposition 9 for the energy momentum tensor of $f_{\mu \nu}$ then the stationary Gödel type metrics (1) with constant $f^{2}$ satisfy the Einstein field equation with a perfect fluid distribution

$$
G_{\mu \nu}=\frac{1}{2}\left(f^{2}+r_{2}\right) u_{\mu} u_{\nu}+\frac{1}{8} f^{2} g_{\mu \nu},
$$

where $r_{2}$ is the Ricci scalar corresponding to the two dimensional metric tensor $h_{\mu \nu}$. Energy density and the pressure of the fluid are respectively given by 


$$
p=\frac{1}{8} f^{2}, \quad \rho=\frac{3}{8} f^{2}+\frac{1}{2} r_{2}
$$

To have some specific solutions we need a coordinate chart. For this purpose let us now consider the metric in polar coordinates (geodesic polar coordinates).

Proposition 11. Without loosing any generality we can write the metric given in (21)in polar coordinates so that the two dimensional part the coordinate curves are orthogonal

$$
d s^{2}=m^{2} d r^{2}+n^{2} d \theta^{2}-\left(u_{0} d t+u_{1} d r+u_{2} d \theta\right)^{2},
$$

where $u_{\mu}=\left(u_{0}, u_{1}, u_{2}\right)$. Here $u_{0}$ is a constant, $x^{\mu}=(t, r, \theta)$, the functions $m, n, u_{1}$ and $u_{2}$ depend on $r$ and $\theta$. The only field equation (23) reduces to a single equation

$$
u_{1, \theta}=u_{2, r}+2 w m n
$$

which is equivalent to $f^{2}=8 w^{2}=$ constant. As a conclusion, for any 3 dimensional metric $g_{\mu \nu}$ with $g_{00}$ is a constant, Eq.(31) solves the stationary Einstein-Perfect fluid equations where the pressure and energy density are given in (29)

\section{Gödel Type Metrics in Topologically Mas- sive Theory}

Topologically Massive Gravity (TMG) equations found by Deser, Jackiw and Templeton (DJT) [8] with a cosmological constant are given as follows.

$$
G_{\nu}^{\mu}+\frac{1}{\mu} C_{\nu}^{\mu}=\lambda \delta_{\nu}^{\mu}
$$

Here $G_{\mu \nu}$ and $R_{\mu \nu}$ are the Einstein and Ricci tensors respectively and $C_{\nu}^{\mu}$ is the Cotton tensor which is given by

$$
C_{\nu}^{\mu}=\eta^{\mu \beta \alpha} \nabla_{\alpha}\left(R_{\nu \beta}-\frac{1}{4} R g_{\nu \beta}\right)
$$


The constants $\mu$ and $\lambda$ are respectively the DJT parameter and the cosmological constant.

To solve the DJT field equations some time ago we have introduced a method [1]. In this method we start with Einstein's equations with a perfect fluid source

$$
G_{\mu \nu}=T_{\mu \nu}
$$

with

$$
T_{\mu \nu}=(p+\rho) u_{\mu} u_{\nu}+p g_{\mu \nu}
$$

where the fluid equations are obtained through the conservation equation $\nabla_{\mu} T^{\mu \nu}=0, p$ and $\rho$ are respectively the pressure and energy density of the fluid and $u_{\mu}$ is the fluid's timelike unit four velocity vector , i.e, $u^{\mu} u_{\mu}=-1$. We have the following result which was reported previously [1]

Proposition 12: If $p, \rho$ are constants and

$$
\nabla_{\mu} u_{\nu}=\frac{\mu}{3} \eta_{\mu \nu \alpha} u^{\alpha}
$$

then any solution of the Einstein equations $G_{\mu \nu}=T_{\mu \nu}$ with a perfect fluid distribution is also a solution of the TMG (32) with a cosmological constant $\lambda=\frac{2 p-\rho}{3}$ and

$$
p=\frac{\mu^{2}}{9}, \quad \rho=\frac{\mu^{2}-9 \lambda_{0}}{3}
$$

where $\lambda_{0}=\lambda+\frac{\mu^{2}}{27}$.

If the Ricci scalar $r_{2}$ of the metric $h_{\mu \nu}$ is a constant then as a consequence of the Proposition 12 any Gödel type of metrics solve the DJT equations

Proposition 13: Stationary Gödel type metrics in three dimensions with constant $f^{2}$, see Proposition 10, solve also the TMG field equations if the two dimensional background space is of constant Gaussian curvature (or $r_{2}=$ constant) and

$$
\mu=3 w, \quad r_{2}=-2\left(w^{2}+3 \lambda\right)
$$


Eq.(38) implies that the two dimensional geometry with the metric $h_{\mu \nu}$ is flat if $\lambda_{0}=0$ As an application of the above Proposition 14 let us consider the following solution of the TMG [1]

Proposition 14. The following metric solves the Topologically Massive Gravity equations exactly

$$
\begin{aligned}
d s^{2} & =-a_{0} d t^{2}+2 q d t d \theta+\frac{-q^{2}+h^{2} \psi}{a_{0}} d \theta^{2}+\frac{1}{\psi} d r^{2} \\
& \left.=-\left(\sqrt{a_{0}} d t-\frac{q}{\sqrt{a_{0}}} d \theta\right)\right)^{2}+\frac{h^{2} \psi}{a_{0}} d \theta^{2}+\frac{1}{\psi} d r^{2}
\end{aligned}
$$

where $u_{0}=\sqrt{a_{0}}, u_{1}=0$ and $u_{2}=-\frac{q}{\sqrt{a_{0}}}$ and

$$
\begin{aligned}
\psi & =b_{0}+\frac{b_{1}}{r^{2}}+\frac{3 \lambda_{0}}{4} r^{2} \\
q & =c_{0}+\frac{e_{0} \mu}{3} r^{2} \\
h & =e_{0} r, \quad \lambda_{0}=\lambda+\frac{\mu^{2}}{27}
\end{aligned}
$$

where $a_{0}, b_{0}, b_{1}, c_{0}$ and $e_{0}$ are arbitrary constants.

The above solution is very special. All the metric functions depend only on the radial coordinate $r$. We may call this solution as stationary spherically symmetric (solutions not depending on the angular coordinate $\theta$ ) Gödel type metrics.

Remark 1. In the study of the black holes solutions in topologically massive gravity, Ait Moussa et al [2] considered the solution of Vuorio [9] which is given by

$$
d s^{2}=-[d \tilde{t}-(2 \cosh \sigma+\tilde{w}) d \tilde{\varphi}]^{2}+d \sigma^{2}+\sinh ^{2} \sigma d \tilde{\varphi}^{2} .
$$

where $\tilde{w}$ is a constant. This solution is a special case of our solution (39) with $\lambda=0, \mu=3$ and

$$
b_{0}=-2, b_{1}=0, e_{0}=1 / 2, a_{0}=1, c_{0}=\tilde{w}-2
$$


The transformation links our solution to the Vuorio solution is given as $t=$ $\tilde{t}, \theta=\tilde{\varphi}, r^{2}=4(1+\cosh \sigma)$. Our analysis shows that the Vuorio solution is also of Gödel type. Hence one may use a similar analytic continuation used in [2] to our solution (39) with $\lambda \neq 0, \mu \neq 3$ to convert it to a black hole solution of TMG. We remark [10] also that the solution given in (39) is equivalent to the solution (3.13) of Ait Moussa et al [3].

Now we will show that, using Proposition 10 it is easy to generalize the above spherically symmetric solution of TMG. Our solution (39) given above has two dimensional space with metric

$$
d s_{2}^{2}=\frac{h^{2} \psi}{a_{0}} d \theta^{2}+\frac{1}{\psi} d r^{2}
$$

where the Gaussian curvature is found as $K=3 \lambda_{0}=3 \lambda+w^{2}$ which is a constant. Ricci scalar $r_{2}$ and the Gaussian curvature $K$ are related by $r_{2}=2 K$. To write the above exact solution of the TMG compatible with the notation in Proposition 10 we get the following identifications

$$
\begin{array}{r}
m=\frac{1}{\sqrt{\psi}}, \quad n=\frac{h \sqrt{\psi}}{\sqrt{a_{0}}} \\
u_{1}=0, \quad u_{2}=-\frac{1}{\sqrt{a_{0}}}\left(c_{0}+w e_{0} r^{2}\right) .
\end{array}
$$

where $\psi$ and $h$ are defined in Proposition 15 .

To generalize the above solution (39) we leave the two dimensional part (42) the same, i.e., letting

$$
m=\frac{1}{\sqrt{\psi}}, \quad n=\frac{h \sqrt{\psi}}{\sqrt{a_{0}}}
$$

and take the most general solution of Eq.(31), i.e.,

$$
u_{1, \theta}=u_{2, r}+2 w \frac{e_{0}}{\sqrt{a_{0}}} r
$$

We solve $u_{2}$ from this equation as

$$
u_{2}=-\frac{1}{\sqrt{a_{0}}}\left(c_{0}+w e_{0} r^{2}\right)+\int^{r}\left(u_{1, \theta}\right) d r
$$


where $c_{0}$ is arbitrary constant and $u_{1}$ is left free. Hence the metric

$$
d s^{2}=m^{2} d r^{2}+n^{2} d \theta^{2}-\left(u_{0} d t+u_{1} d r+u_{2} d \theta\right)^{2}
$$

with $m, n$, and $u_{2}$ given above (45) and (47) respectively, solve the TMG exactly. Here $u_{1}(r, \theta)$ is left arbitrary which was taken to be zero in our solution (39). Hence we have the following result:

Proposition 15. We obtain the most general stationary solution of Topologically Massive Gravity when $g_{00}$ is a constant. The solution is given in Gödel type where $u_{0}=\sqrt{a_{0}}, u_{1}$ is an arbitrary function of $r$ and $\theta, u_{2}$ is given in (47) and the two dimensional metric is given in (42) with constant Gaussian curvature $K=3 \lambda_{0}$. This solution generalizes our solution presented in [1]

\section{$5 \quad$ Ricci and Cotton Flows}

In this section we shall assume that the Gaussian curvature $K$ of the two dimensional space with metric $h_{\mu \nu}$ is a constant. From Proposition 10 we have the Ricci tensor of a stationary Gödel type metrics

$$
R_{\mu \nu}=\frac{1}{2}\left(f^{2}+r_{2}\right) u_{\mu} u_{\nu}+\frac{1}{2}\left(r_{2}+\frac{1}{2} f^{2}\right) g_{\mu \nu}
$$

where $r_{2}=2 K$ is the Ricci scalar corresponding to the two dimensional metric tensor $h_{\mu \nu}$. Then we have an exact solution of the Ricci flow equation

Proposition 16. Let $(M, g)$ be the stationary Gödel type spacetime with $f^{2}=8 w^{2}$ constant as in Proposition 10. Then Ricci flow equation [11]

$$
\frac{\partial g_{\mu \nu}}{\partial s}=\xi R_{\mu \nu}
$$

where $s$ is the flow parameter and $\xi$ is an arbitrary constant, has an exact solution if

$$
\begin{aligned}
\frac{\partial u_{\mu}}{\partial s} & =-\xi w^{2} u_{\mu} \\
\frac{\partial h_{\mu \nu}}{\partial s} & =-\xi\left(K-2 w^{2}\right) h_{\mu \nu} .
\end{aligned}
$$


The above flow equations (51) and (52) are solved exactly by playing with the constants $b_{0}, c_{0}, \lambda_{0}$, and $e_{0}$. As an example $u_{0}$ (or $a_{0}$ ) has the following behavior under the this flow

$$
u_{0}=u_{0}(0) e^{-\xi w^{2} s}
$$

where $u_{0}(0)$ is an arbitrary constant. On the other hand taking the trace of both sides of $(50)$ we obtain that $u_{0}=u_{0}(0) e^{-\xi\left(w^{2}+K\right) s}$. Hence comparing with (53) we get $K=0$.

Proposition 17. The Ricci flow equations have Gödel type of metrics as exact solutions only when the two dimensional space is a space of zero curvature

Proposition 18 The Cotton tensor for stationary Gödel type metrics take the following simple form

$$
C_{\nu}^{\mu}=-w(p+\rho)\left(\delta_{\nu}^{\mu}+3 u^{\mu} u_{\nu}\right)
$$

Hence the spacetime geometry $(M, g)$ is conformally flat if $\lambda=\frac{\mu^{2}}{9}$. The Cotton flow equation

$$
\frac{\partial g_{\mu \nu}}{\partial s}=\zeta C_{\mu \nu}
$$

where $\zeta$ is a constant and $s$ is the flow parameter. These equations were recently used by [12]. Here we show that Gödel type metrics solve exactly the Cotton flow equations (55) only when the Cotton tensor vanishes. For $C_{\mu \nu}$ given above we have

Proposition 19.Let $(M, g)$ be the stationary Gödel type spacetime with $f^{2}=$ $8 w^{2}$ constant as in Proposition 10. Then Cotton flow equations (55) lead to the following flow equations for $h_{\mu \nu}$ and $u_{\mu}$

$$
\begin{aligned}
\frac{\partial u_{\mu}}{\partial s} & =-k u_{\mu}, \\
\frac{\partial h_{\mu \nu}}{\partial s} & =-k h_{\mu \nu} .
\end{aligned}
$$


where $k=\zeta w(p+\rho)$. Again the above flow equations (56) and (57) are solved exactly by playing with the constants $b_{0}, c_{0}, \lambda_{0}$, and $e_{0}$. As an example $u_{0}$ (or $a_{0}$ ) has the following behavior under the Cotton flow

$$
u_{0}=u_{0}(0) e^{-k s}
$$

where $u_{0}(0)$ is an arbitrary constant. On the other hand taking the trace of both sides of (55) we obtain $u_{0}$ is also constant with respect to the flow parameter $s$. Comparing this with (58) we obtain $k=0$.

Proposition 20. Let $(M, g)$ be the stationary Gödel type spacetime with $f^{2}=$ $8 w^{2}$ constant as in Proposition 10. Then Cotton flow equations (55) have exact solutions only when the $k=0$, but this means that the Cotton tensor vanishes.

\section{Gödel Type Metrics in Higher Curvature Theories}

In three dimensions when the stationary Gödel type metrics with constant $f^{2}$ have further nice properties. It is possible to show that the tensors $u_{\mu}$, $f_{\mu \nu}$ and $g_{\mu \nu}$ satisfy the following tensorial algebra.

Proposition 21. Let $D=3$ and the metric of spacetime be Gödel type with constant $f^{2}$. Let the two dimensional space with metric $h_{\mu \nu}$ be a space of constant Gaussian curvature. Then we have the following closed differential algebra of the tensors $u_{\mu}, f_{\mu \nu}$ and $g_{\mu \nu}$

$$
\begin{aligned}
\nabla_{\mu} u_{\alpha} & =\frac{1}{2} f_{\mu \alpha} \\
f_{\mu \alpha} & =w \epsilon_{\mu \alpha \sigma} u^{\sigma} \\
\nabla_{\alpha} f_{\mu \beta} & =\frac{1}{2} w^{2}\left(g_{\mu \alpha} u_{\beta}-g_{\beta \alpha} u_{\mu}\right), \\
\nabla_{\mu} g_{\alpha \beta} & =0 .
\end{aligned}
$$

From the previous Propositions (in particular Proposition 10) we can deduce that The Ricci, Einstein , curvature tensors and their contractions 
at any order will be the linear sum the tensors $u_{\mu} u_{\nu}$ and $g_{\mu \nu}$. Hence the tensor differential algebra introduced in (59)-62) is effective to show that the gravitational field equations, for any gravitational action. are given as follows

$$
\begin{aligned}
G_{\mu \nu} & =A g_{\mu \nu}+B u_{\mu} u_{\nu} \\
\nabla_{\mu} f^{\mu \nu} & =C u^{\nu}
\end{aligned}
$$

where $A, B$ and $C$ are constants depending upon the theory. This leads to the following result:

Proposition 22. Let the action of gravitation contains all possible combinations of Ricci, curvature and the antisymmetric tensor $F_{\mu \nu}$ and their covariant derivatives at any order. Then the tensor differential algebra introduced in (59)-(62) is effective to show that the gravitational field equations are solved when the metric is the stationary Gödel type metrics with constant $f^{2}$ and the two dimensional background is a space of constant Gaussian curvature $K$, and $F_{\mu \nu}=f_{\mu \nu}$ at all orders of the string tension parameter.

\section{Conclusion}

We showed that the metric of any three dimensional stationary spacetime with $g_{00}$ constant satisfies the Einstein-perfect fluid equations. The only differential equation to be solved is a first order partial differential for the components of the fluid velocity vector field. We then showed that in this spacetime symmetry with $g_{00}$ constant we find the most general solution of the TMG. This solution generalizes our previous solution [1] (Proposition 15). We showed that stationary Gödel type metrics constitute a very simple

solution of the Ricci flow equations an do not solve the Cotton flow equations (Propositions 19 and 20). Finally we discussed the possibility that the stationary Gödel type metrics form a solution of the low energy limit of string theory with the most possible interactions of curvature and antisymmetric field $F_{\mu \nu}$ (Proposition 22). 
I would like to thank Professors Gerard Clement and Atalay Karasu for reading the manuscript and constructive comments. This work is partially supported by the Scientific and Technological Research Council of Turkey (TUBITAK) and Turkish Academy of Sciences (TUBA).

\section{References}

[1] M. Gürses, Class. Quantum Grav. 11, 2585 (1994).

[2] K.A. Moussa, G. Clement, and C. Leygnac, Class. Quantum Grav. 20,L277-L283(2003).

[3] K.A. Moussa, G. Clement, H. Guennoune and C. Leygnac, Phys.Rev. D78, 064065 (2008).

[4] J.D. Barrow, D.J. Shaw, and C.G. Tsagas, Class. Quantum Grav. 23, 5291-5322 (2006).

[5] M. Gürses, A. Karasu and Ö. Sarıoglu, Class. Quantum Grav. 22, 1527-1543 (2005).

[6] M. Gürses and Ö. Sarıg̃̃lu, Class. Quantum Grav. 22, 4699(2005).

[7] R.J. Gleiser, M. Gürses, A. Karasu and Ö. Sarıõ̃lu, Class. Quantum Grav. 23, 2653 (2006).

[8] S. Deser, R. Jackiw, and S. Templeton, Phys. Rev. Lett. 48, 975 (1982); Ann. Phys., NY 140, 372 (1982).

[9] I. Vuorio, Phys. Lett. B163, 91 (1985); R. Percacci, P. Sodano, and I. Vuorio, Ann. Phys. NY, 176, 344 (1987).

[10] G. Clement, Private Communication.

[11] R.S. Hamilton, J. Differ. Geom. 17, 255 (1982).

[12] A. U. Kişisel, Ö. Sarıog̃lu, and B. Tekin, Class. Quantum. Grav. 25, 165019 (2008). 